\title{
Factor Predicting Cholestasis in Post Modified Right Lobe Hepatectomy in the Era of Standardized Donor Selection Protocols
}

\author{
Verma S*, Naganathan SK, Dey R, Agarwal S and Gupta S \\ Center for Liver and Biliary Sciences, Max Super Speciality Hospital, India
}

*Corresponding author: Dr. Sapana Verma, MD PhD, Center for Liver and Biliary Sciences, Max Super Speciality Hospital, Saket 1, Press Enclave Road, Saket, New Delhi- 110 017, India, Tel: +91-9560479231; +91-2001-40633549; Email: sapana_21@hotmail.com

\section{Research article \\ Volume 4 Issue 1}

Received Date: March 05, 2020

Published Date: April 20, 2020

DOI: $10.23880 /$ ijtps-16000140

\section{Abstract}

Inspite of increase in number of living related liver transplantation (LRLT), right lobe donation continues to be high risk surgery. Of all the donor mortality reported so far post-operative liver failure secondary to small remnant remains high. Hence, we analysed our data to look for risk factors for cholestasis following post modified right lobe (MRL) donor hepatectomy.386 donors who underwent MRL donor hepatectomy from Feb 2017 to July 2019 were included in the study. We analysed data based on 2 cut off values of total bilirubin (Tbil), one based on median peak bilirubin $(2.3 \mathrm{mg} / \mathrm{dL}$ ) of study cohort and another $5 \mathrm{mg} / \mathrm{dL}$ which is considered as average high cut off of Tbil at our centre. With $2.3 \mathrm{mg} / \mathrm{dL}$ cut off, males, spouses, remnant $\%$, graft weight, INR were significant $(\mathrm{P}<0.05)$. On analysis with Tbil $5 \mathrm{mg} / \mathrm{dL}$ cut off, male gender, blood group 0 , Body mass index (BMI), INR were significant $(\mathrm{P}<0.05)$.0n multivariate analysis male donors alone tend to be high risk for cholestasis in both group and Blood group 0 in the Tbil $>5$ cut off group. Male gender and blood group 0 were significant risk factor for post MRL donor hepatectomy cholestasis.

Keywords: Liver Donor; Transplantation; Hepatectomy

Abbreviations: LDLT: Liver Donor Liver Transplantation; MRL: Modified Right Lobe; TB: Total Bilirubin; INR: International Normalisation Ratio; POD: Post-Operative Day; BMI: Body Mass Index; DDLT: Deceased Donor Liver Transplant; PP: Portal Pressure; OR: Odds Ratio; CI: Confidence Interval; LAI: Liver Attenuation Index.

\section{Introduction}

The first liver transplantation (LT) was performed by Thomas E Starzl more than six decades ago and it remains the gold standard treatment for end stage liver disease. Deceased donor LT (DDLT) is the operation of choice for these patient however disparity between demand and supply of organ, living related liver transplant (LRLT) was introduced [1]. Due to the enduring organ shortage, living donor liver transplantation has been a valuable treatment strategy for advanced liver disease patients for over 20 years [2]. LRLT was started with children and left lateral segments were resected when the deceased donor and non-heart beating donor didn't fulfil the demand of the organ in those group [3]. LDLT has several advantages several such as shorter waiting time, shorter warm ischemia time cold ischemia time and it is an ideal solution, especially when no other grafts are available and the surgery is urgent [4].

In spite of all the benefits, the safety of living donor remains controversial and therefore, donor safety is central 


\section{International Journal of Transplantation \& Plastic Surgery}

to LDLT program. As the application of LDLT extended from children to adults, and from using the left liver graft to the right liver graft, the dilemma between recipient success and donor risk came to the spotlight. With Improved surgical techniques and better understanding of liver regeneration and finer imaging more complicated donor hepatectecomies are done in the LRLT program [5]. Despite, of so many hepatectomies done so far there is a definite risk of donor mortality even today [6]. Since the adoption of the right lobe liver for LDLT, concerns about a perfectly healthy donor receiving a major hepatectomy have emerged; though the risk is low, but almost definite [7]. Studies have shown that Right-hepatectomy or extended right-hepatectomy have significantly more disadvantageous impact on major complications [8]. We therefore further in the present study identified and evaluated perioperative risk factors for cholestasis post MRL donor hepatectomy.

\section{Patients and Methods}

We enrolled 386 live donor who underwent right lobe donation from the period of February 2017 to July 2019 at our centre. All donors were healthy adults and Kin to the recipient. None of the transplant donors were from a vulnerable population and all donors or next of kin provided written informed consent that was freely given.

\section{Donor Evaluation}

All live donor applied for live donation voluntarily without any financial benefit. Donors age more than 18 and less than 60 years was considered as fit for donation. Identical blood group was preferred but incompatible group also accepted. No physical or mental morbidity, which might impact the tolerance and awareness of the procedure, should be found in donors.

Donor work up was proceeded as per Our Evaluation Algorithm for Selecting Living Donor Candidate for LT as shown in Table 1. After completion of blood work up we proceeded for 3 tesla MRI done to visualise the biliary anatomy and fat assessment was also done by Proton density fat fraction technique. When the fat was more than $17 \%$ on imaging, we did a liver biopsy. If biopsy shows more than 30 $\%$ then they are placed on weight loss protocol until there was correction of fat fraction on imaging. CECT Computed Tomography (CT) scan done with 126 slicer CT Philips system with timings of Vascular Anatomy was assessed along with Remnant Assessment (GE, Vicar software). At least 30\% including caudate lobe with MHV for volumetric measurement was performed to evaluate graft size and the size of remnant donor liver. Remnant volume of $<30 \%$ of the whole liver volume was regarded as optimal safety margin for donors.

\begin{tabular}{|c|}
\hline Step 1 \\
\hline Haematology \\
\hline Blood Grouping \\
\hline Complete Blood Count \\
\hline PT, PTTK \\
\hline Biochemistry \\
\hline Liver Function Tests \\
\hline Urea, Creatinine \\
\hline Sodium, Potassium \\
\hline Random Blood Sugar \\
\hline Lipid Profile, HBa1c \\
\hline TSH, T3, T4 \\
\hline Serology \\
\hline HBsAg \\
\hline HIV I \& II \\
\hline Anti HCV \\
\hline HBc core $\mathrm{Ab}$ \\
\hline Anti HBsAb titre \\
\hline CMVIgG \\
\hline G6PD \\
\hline Clinical Pathology \\
\hline Urine R/E \\
\hline Step 2 \\
\hline MRCP \\
\hline Step 3 \\
\hline $\begin{array}{c}\text { CECT liver triple phase angiogram (1. LAI, 2. Volumetry } 3 . \\
\text { Liver Angiography) }\end{array}$ \\
\hline HLA Class I \&II \& Cross match \\
\hline Step 4 \\
\hline Chest X-Ray \\
\hline Cardiology \\
\hline ECG \\
\hline PFT \\
\hline 2 D ECHO / Stress echo (if $>40$ yrs ). \\
\hline Gynaecology Clearance \\
\hline Only for female donors \\
\hline (USG ABDOMEN AND PELVIS). \\
\hline PAPS Smear ( If Married)_. \\
\hline Breast Package ( age more than 35 yrs)_. \\
\hline Psychiatry Test \\
\hline
\end{tabular}

Table 1: Our Evaluation Algorithm for Selecting Living Donor Candidate for LT. 


\section{International Journal of Transplantation \& Plastic Surgery}

\section{Operative Techniques}

A Makuchi incision was routinely applied. Liver remnant was reassessed by senior surgeons.

Intraoperative cholangiogram (IOC) was done to reconfirm the biliary anatomy. Then portal pressure (PP) and Middle Hepatic Vein (MHV) congestion measurement are done. If PP more than 3 then it was contraindication for modified right lobe, surgical technique is modified or else the donor operation is abandoned. If no abnormality was found, the surgery was continued by parenchyma transection with Cavitron Ultrasonic Suction and Aspirator (CUSA). The transaction line was determined by hepatic vascular occlusion. Veins $(>5 \mathrm{~mm})$ were temporarily clippedwith titanium clip and were used for further reconstruction in the recipient. After total dissociation of the right lobe, the right hepatic artery, right portal trunk, and right hepatic vein were interdicted and transected in an orderly manner. Heparinisation was done before ligating the hepatic veins, the haemorrhage and bile leakage were paired with 5-0 prolene and 6-0 PDS (polydioxanone), respectively, over the transected surface. IOC was repeated to assess the remaining biliary system at the end of the operation. The weight of the graft was measured at the back table and documented into our database. Intra-peritoneal sub-hepatic tube was placed in all cases.

\section{Postoperative Treatment and Follow-up}

All donors were extubated on table. Arterial blood gas (ABG) analysis was done as lactate is a good marker of patient recovery. Donors were transferred to Intensive Care Unit (ICU) for liver transplantation right after the procedure. ABG was done 4 hourly. Intravenous antibiotics, Proton pump inhibitors, intravenous analgesics were given and continued until POD2. Liver doppler was done on POD 1. Once donors were stable, they were moved to clinical ward (CW). Intra peritoneal sub-hepatic drains were removed on POD4 once drain output was low and sero hemorrahgic. Donors were given abdominal belt for two weeks. The total hospital Length of Stay (LOS) was calculated as time admitted to the ICU to the time of discharge. All the donors were discharged with normalised Tbil and INR, with healthy wound and normal lifestyle. They were prescribed oral analgesics to if required and was called for follow up after 3 days. At outpatient department they were advised for basic Complete blood count, Liver function test, Kidney function to see the general wellbeing. After that they were not called for follow up until they had any problems.

\section{Statistical Analysis}

Follow up was continued until October 2019.To identify risk factor for cholestasis of donors variables concerning living donors were assessed by univariate analyses using the chi-square test or Fisher's exact test where appropriate were performed to compare categorical variables, and the Wilcoxon rank sum test was used to compare continuous variables. A logistic model was applied for estimation of odds ratio (OR) associated with post-operative donor cholestasis. Statistical analyses were performed using JMP statistical software, version Genomics 13 (SAS Institute, Cary, NC). All $\mathrm{P}$ values presented are two-sided. $\mathrm{P}$ values of $<0.05$ were considered statistically significant.

\section{Results}

\section{Demographics and Operative Variables of the Living Liver Donors}

Donor demographics and operative variables were analysed (data not shown). Of 386 donors, 178 (46.11\%) were females and $208(53.88 \%)$ were males. The mean age was 33.04 years and mean BMI was 25.5. The mean LAI was 8.34 and mean \pm SD right lobe volume without MHV was $831.86 \pm 168.72$. The mean remnant percent was $35.7 \%$. Intraoperative parameters were as the mean congestion area was $38.8 \%$ with mean actual graft weight $781.2 \pm 142.3 \mathrm{gm}$.

\section{Postoperative Cholestasis}

We further looked for risk factors for cholestasis. Risk factors were analysed based on two cut off values of Tbil as mentioned above. On analysis the median Peak Bilirubin was $2.3 \mathrm{mg} / \mathrm{dL}$. Of the 286 living donors, $185 \mathrm{had}$ more than 2.3 $\mathrm{mg} / \mathrm{dL}$ peak bilirubin. We analysed several perioperative variables to find out the risk factors for donor cholestasis in between the two groups. Further we also divided cholestasis group with the cut of peak Tbil as $5 \mathrm{mg} / \mathrm{dL}$. Of the 386 living donors, 19 had Tbil more than $5 \mathrm{mg} / \mathrm{dL}$.

\section{Risk Factors for Post MRL Hepatectomy Cholestasis}

We analysed several risk factors for development of cholestasis in both groups. When we took the Tbil cut off as $2.3 \mathrm{mg} / \mathrm{dL}$, on univariate analysis we found that male gender had higher chance of developing cholestasis $(45.27 \%$ vs $63.24 \%$, P value $=0.0004)$, and less remnant $\%$ (36.38 \pm 4.76 vs $34.96 \pm 4.55$, $\mathrm{P}$ value $=0.001$ ), was found to be significant risk factors (Table 2). Similarly, when we compared the variables with the cut off as $5 \mathrm{mg} / \mathrm{dL}$, male gender $(52.59 \%$ vs $78.95 \%$, P value $=0.024)$, Blood group 0 (51.5 \% vs 78.95 $\%$, P value $=0.019)$, less BMI (25.69 \pm 4.59 vs $23.14 \pm 2.45$, $\mathrm{P}$ value $=0.005$ ) were significant risk factors between 2 groups. (Table 3). 


\section{International Journal of Transplantation \& Plastic Surgery}

\begin{tabular}{|c|c|c|c|}
\hline Variables & $\begin{array}{c}<2.3 \\
(n=201)\end{array}$ & $\begin{array}{c}\geq 2.3 \\
(n=285)\end{array}$ & $P$ value \\
\hline Age & $33.04 \pm 10.8$ & $33.04 \pm 10.18$ & 0.81 \\
\hline Gender & & & 0.0004 \\
\hline male & $91(45.27)$ & $117(63.24)$ & \\
\hline female & $110(54.73)$ & $68(36.76)$ & \\
\hline Blood type & & & 0.57 \\
\hline Non 0 & $92(45.77)$ & $90(48.65)$ & \\
\hline 0 & $109(54.23)$ & $95(51.35)$ & \\
\hline Relation & & & 0.019 \\
\hline Not Kin & $0(0)$ & $5(2.7)$ & \\
\hline Kin & $201(100)$ & $180(97.3)$ & \\
\hline BMI & $25.4 \pm 4.95$ & $25.6 \pm 4.07$ & 0.41 \\
\hline LAI & $8.8 \pm 14.91$ & $7.7 \pm 6.34$ & 0.22 \\
\hline $\begin{array}{l}\text { Rt. liver volume with CT } \\
\text { (cm3) }\end{array}$ & $826.84 \pm 168.38$ & $837.28 \pm 169.38$ & 0.41 \\
\hline MPV mm & $11.8 \pm 1.79$ & $12.6 \pm 8.54$ & 0.88 \\
\hline RPV mm & $10.35 \pm 1.77$ & $10.61 \pm 1.53$ & 0.26 \\
\hline LPV $\mathrm{mm}$ & $8.68 \pm 1.54$ & $8.89 \pm 1.85$ & 0.5 \\
\hline RHV mm & $10.13 \pm 2.21$ & $9.71 \pm 2.26$ & 0.11 \\
\hline Remnant (\%) & $36.38 \pm 4.76$ & $34.96 \pm 4.55$ & 0.001 \\
\hline \multicolumn{4}{|l|}{ Operative variable } \\
\hline Congestion area & $39.2 \pm 11.7$ & $38.4 \pm 10.9$ & 0.8 \\
\hline Actual graft weight & $766.5 \pm 143.2$ & $797.1 \pm 140$ & 0.04 \\
\hline CPP (Pre clamp) & $3.37 \pm 1.82$ & $3.59 \pm 1.83$ & 0.24 \\
\hline CPP (Post clamp) & $4.25 \pm 2$ & $4.53 \pm 2.01$ & 0.26 \\
\hline CPP (Post transaction) & $4.21 \pm 2.05$ & $4.32 \pm 1.92$ & 0.48 \\
\hline Pressure difference & $0.91 \pm 0.81$ & $0.91 \pm 0.81$ & 0.78 \\
\hline No of Hepatic artery & & & 0.28 \\
\hline One & $179(89.05)$ & $158(85.41)$ & \\
\hline More than one & $22(10.95)$ & 27 (14.59) & \\
\hline No of Bile ducts & & & 0.56 \\
\hline One & $155(77.11)$ & $138(74.59)$ & \\
\hline More than one & $46(22.89)$ & $47(25.41)$ & \\
\hline Total Bilirubin (D1) & $1.53 \pm 0.42$ & $3.10 \pm 1.03$ & $<0.0001$ \\
\hline INR (D1) & $1.65 \pm 0.25$ & $1.69 \pm 0.27$ & 0.23 \\
\hline Platelets (D1) & $228102 \pm 60775$ & $229282 \pm 132363$ & 0.24 \\
\hline Total Bilirubin (D3) & $1.47 \pm 0.36$ & $2.86 \pm 1.31$ & $<0.0001$ \\
\hline INR (D3) & $1.61 \pm 0.3$ & $1.7 \pm 0.4$ & 0.04 \\
\hline Platelets (D3) & $200000 \pm 51938$ & $185783 \pm 43132$ & 0.11 \\
\hline Total Bilirubin (D5) & $1.54 \pm 0.57$ & $3.09 \pm 1.56$ & $<0.0001$ \\
\hline
\end{tabular}




\section{International Journal of Transplantation \& Plastic Surgery}

\begin{tabular}{|c|c|c|c|}
\hline INR (D5) & $1.45 \pm 0.25$ & $1.35 \pm 0.24$ & 0.32 \\
\hline Platelets (D5) & $207750 \pm 59150.5$ & $212167 \pm 74334.9$ & 0.82 \\
\hline Peak Tbil & $1.64 \pm 0.39$ & $3.51 \pm 1.30$ & 0.0001 \\
\hline Peak INR & $1.70 \pm 0.28$ & $1.78 \pm 0.38$ & 0.43 \\
\hline POD to peak Tbil & $2.20 \pm 2.53$ & $1.96 \pm 1.65$ & 0.02 \\
\hline POD to peak INR & $2.04 \pm 2.51$ & $1.80 \pm 1.22$ & $<0.0001$ \\
\hline POD to Normal Tbil & $6.35 \pm 4.52$ & $8.83 \pm 3.42$ & 0.54 \\
\hline Hospital stay & $10.56 \pm 1.92$ & $10.76 \pm 2.12$ & \\
\hline
\end{tabular}

Table 2: Characteristics and Perioperative Data of MLR Donors with Tbil cut off $>2.3$.

Categorical variable are presented as number (\%) while continuous variable are presented as mean $\pm \mathrm{SD}^{*}$.

Pearson chi-square test/ Wilcoxon test, A difference was considered significant if p-value was $<0.05$.

BMI, body mass index, LAI, liver attenuation index, MPV, main portal vein, RPV, right portal vein, LPV, left portal vein, RHV, right hepatic vein, CPP, corrected portal pressure, INR, international normalization ratio, Tbil, Total bilirubin, POD, post-operative day.

\begin{tabular}{|c|c|c|c|}
\hline Variables & $\begin{array}{c}<5.0 \\
(n=367)\end{array}$ & $\begin{array}{c}\geq 5.0 \\
(n=19)\end{array}$ & $P$ value \\
\hline Age (years) & $33.01 \pm 10.65$ & $33.73 \pm 8.11$ & 0.49 \\
\hline Gender & & & 0.024 \\
\hline male & $193(52.59)$ & $15(78.95)$ & \\
\hline female & $174(45.08)$ & $4(21.05)$ & \\
\hline Blood type & & & 0.019 \\
\hline Non 0 & $178(48.5)$ & $4(21.04)$ & \\
\hline 0 & $189(51.5)$ & $15(78.95)$ & \\
\hline Relation & & & 0.11 \\
\hline Not Kin & $4(1.09)$ & $1(5.29)$ & \\
\hline Kin & $363(98.91)$ & $18(91.74)$ & \\
\hline BMI & $25.69 \pm 4.59$ & $23.14 \pm 2.45$ & 0.005 \\
\hline LAI & $8.39 \pm 11.79$ & $7.29 \pm 7.53$ & 0.52 \\
\hline Rt. liver volume with CT (cm3) & $830.4 \pm 170$ & $858.6 \pm 141.1$ & 0.35 \\
\hline MPV mm & $11.87 \pm 1.63$ & $18.33 \pm 25.16$ & 0.04 \\
\hline RPV mm & $10.44 \pm 1.67$ & $11.08 \pm 1.38$ & 0.04 \\
\hline LPV mm & $8.76 \pm 1.71$ & $9.14 \pm 1.64$ & 0.39 \\
\hline RHV & $9.91 \pm 2.23$ & $10.22 \pm 2.50$ & 0.47 \\
\hline Remnant (\%) & $35.6 \pm 4.48$ & $35.8 \pm 8.09$ & 0.35 \\
\hline \multicolumn{4}{|l|}{ Operative variable } \\
\hline Congestion area (\%) & $39.03 \pm 11.31$ & $35.33 \pm 11.42$ & 0.09 \\
\hline Actual graft weight (gm) & $781.13 \pm 142.87$ & $784.4 \pm 135.35$ & 0.88 \\
\hline CPP (Pre clamp) & $3.48 \pm 1.85$ & $3.38 \pm 1.37$ & 0.82 \\
\hline CPP (Post clamp) & $4.39 \pm 3.01$ & $4.36 \pm 1.92$ & 0.81 \\
\hline CPP (Post transaction) & $4.27 \pm 1.99$ & $4.21 \pm 1.93$ & 0.94 \\
\hline Pressure difference & $0.90 \pm 0.81$ & $1.15 \pm 0.76$ & 0.14 \\
\hline No of Hepatic artery & & & 0.67 \\
\hline
\end{tabular}




\section{International Journal of Transplantation \& Plastic Surgery}

\begin{tabular}{|c|c|c|c|}
\hline One & $321(87.47)$ & $16(84.21)$ & $3(15.79)$ \\
\hline More than one & $46(12.53)$ & & 0.81 \\
\hline No of Bile ducts & & $14(73.68)$ & \\
\hline One & $279(76.02)$ & $5(26.52)$ & $<0.0001$ \\
\hline More than one & $88(23.98)$ & $4.92 \pm 1.12$ & 0.07 \\
\hline Total Bilirubin (D1) & $2.14 \pm 0.91$ & $1.75 \pm 0.22$ & 0.56 \\
\hline INR (D1) & $1.66 \pm 0.26$ & $217684 \pm 54562$ & $<0.0001$ \\
\hline Platelets (D1) & $229236 \pm 103313$ & $5.02 \pm 2.39$ & 0.041 \\
\hline Total Bilirubin (D3) & $2.09 \pm 0.82$ & $1.92 \pm 0.62$ & 0.82 \\
\hline INR (D3) & $1.64 \pm 0.33$ & $195182 \pm 59207$ & $<0.0001$ \\
\hline Platelets (D3) & $191264 \pm 46371$ & $5.14 \pm 1.86$ & 0.46 \\
\hline Total Bilirubin (D5) & $2.34 \pm 0.90$ & $1.46 \pm 0.31$ & 0.27 \\
\hline INR (D5) & $1.35 \pm 0.22$ & $180833 \pm 66161$ & $<0.0001$ \\
\hline Platelets (D5) & $219800 \pm 68687$ & $6.45 \pm 1.83$ & 0.0009 \\
\hline POD of Peak Bil & $2.34 \pm 0.93$ & $3.15 \pm 2.06$ & $<0.0001$ \\
\hline POD to N bil & $2.03 \pm 2.15$ & $11.47 \pm 4.11$ & 0.012 \\
\hline Peak INR & $7.34 \pm 4.12$ & $2 \pm 0.57$ & 0.01 \\
\hline POD of Peak INR & $1.72 \pm 0.31$ & $2.15 \pm 1.01$ & 0.32 \\
\hline POD to N INR & $1.91 \pm 2.04$ & $11.68 \pm 4.09$ & \\
\hline Hospital stay & $10.60 \pm 1.84$ & & \\
\hline
\end{tabular}

Table 3: Characteristics and Perioperative Data of MLR Donors with Tbil cut off $>5$.

Categorical variable are presented as number(\%) while continuous variable are presented as mean \pm SD.

*: Pearson chi-square test/ Wilcoxon test ,A difference was considered significant if p-value was $<0.05$.

\section{Risk Factors on Multivariate Analysis}

The multivariate logistic regression analysis identified one independent factor that is male gender that contributed significantly to the differences between patients with and without cholestasis when the the cut of Tbil was $2.3 \mathrm{mg} \%$ (OR 2.14 [confidence interval (CI): 1.23-2.73], $\mathrm{p}=0.006$ respectively) (Table 4). Similarly when we took the Tbil cut off as $5 \mathrm{mg} \%$, we found out that blood group 0 (OR 4.9, (CI): 1.26- 19.8, $\mathrm{p}=0.02$ ) and again male gender (OR 0.85, (CI): 0.76- 0.97, $\mathrm{p}=0.01$ ) (Table 5) was significant contributory for development of major cholestasis after MRL in healthy donor.

\begin{tabular}{|c|c|c|c|c|}
\hline Variables & OR & \multicolumn{2}{|c|}{$95 \%$ CI } & P-value \\
\hline Gender & 2.14 & 1.23 & 2.73 & 0.006 \\
\hline
\end{tabular}

Table 4: Multivariate Analysis of Factors Related to cholestasis when Tbil cut off $>2.3$.

A logistic model was applied for estimation of odds ratio (OR) associated with risk factors for donor cholestasis.

A difference was considered significant if the p-value was $<0.05 \mathrm{CI}$, confidence interval.

\begin{tabular}{|l|l|l|l|l|}
\hline O group & 4.9 & 1.26 & 19.8 & 0.02 \\
\hline BMI & 0.85 & 0.76 & 0.97 & 0.01 \\
\hline
\end{tabular}

Table 5: Multivariate Analysis of Factors Related to Cholestasis when Tbil cut off $>5$.

A logistic model was applied for estimation of odds ratio (OR) associated with risk factors for donor cholestasis. A difference was considered significant if the p-value was $<0.05 \mathrm{CI}$, confidence interval.

\section{Discussions}

LDLT has evolved as a compliment to DDLT because of donor organ shortage. It violates the basic principle of surgery that is primum non nocere. Donor hepatectomy has evolved over years and because of more experience over years and better understanding of anatomy and physiology the complication rates have been brought under control $[4,8,9]$. Many centres have evolved and are doing laparoscopic and robotic donor hepatectomies with minimal complications $[10,11]$. However, this in turn has increased the potential complications even further for the donors as new centres have started attempting and in that learning curve new 


\section{International Journal of Transplantation \& Plastic Surgery}

wave of complications and potential donor morbidity and death are possible. With our personal experience of more than 2800 living donor liver transplantations we are also moving towards minimally invasive donor hepatectomies. In view of high volume and limited donor availabilities our protocols are updated at constant intervals [12]. Many high volume centres now have no anatomical contraindication for donor hepatectomies. Remnant volume assessment still seems to be the achilles heel of safe donor hepatectomy. CT volumetry, MR volumetry, ICG excretion and many other methods are followed. Despite all these imaging modalities, volume assessment by the experienced surgeon is the best modality. There are many instances where the imagings have calculated lesser remnant but on visual analysis found to have good remnant operation were proceeded with and donors have uneventful recovery. In our institute many donors are rejected because of lesser remnants $<30 \%$. Hence it is fine balance of rejecting a donor and saving a recipient. Keeping these factors in mind the policy is to do intraoperative assessment in case of borderline donors before we reject them. We do intraoperative assessment by consensus decision by senior surgeons, portal pressure monitoring before and after clamping the lobar portal veins and assessment of congestion area of the middle hepatic veins. Based on these parameters we try to modify the transection line and avoid damaging the middle vein tributaries and there by not compromise the remnant. Inspite of or in view of all these practical day to day modifications some donors still end up having unusual cholestasis after operation. We therefore analysed various parameters to find out the risk factors related to donor cholestasis. On univariate analysis, we found male gender, blood type 0 , lower BMI, low remnant and lower, day3 INR, POD to peak INR as risk factor for development of cholestasis. Further on multivariate analysis we found male gender, lesser BMI and blood group $\mathrm{O}$ significant risk factor. Males were more at risk of prolonged cholestasis than females. The possibilities seems to be same as that of BMI. Females have lesser lean body mass than males. Role of immunological mechanism of reproductive hormones is again a possibility which may help in quicker liver regeneration than males [13]. 0 positive blood group has some relation with cholestasis. It is a known phenomenon that 0 Positive blood group of mother is associated with cholestasis in neonates [14]. Moreover it may be a biased analytical result that $\mathrm{O}$ blood group is universal donor and hence the number of 0 group donors seems to far outweigh other group donors.

Based on our analysis almost all the hyperbilirubinemias settled without any problems with conservative management. Since we did not have any mortality we could not comment more about it. Further experience or analysis of pooled data of donor deaths will be able to give us a light on the significance of post hepatectomy cholestasis. In conclusion, our study identified the group of donors who are at risk for development of cholestasis which should be vigorously manage to avoid significant donor morbidity. Further studies and larger cohort are required to prove the further basic mechanism behind those risk factors.

\section{References}

1. Smith B (1969) Segmental liver transplantation from a living donor. J Pediatr Surg 4(1): 126-132.

2. Chan SC, Fan ST (2008) Historical perspective of living donor liver transplantation. World J Gastroenterol 14(1): 15-21.

3. Broelsch CE, Whitington PF, Emond JC, Heffron TG, Stevens L, et al. (1991) Liver transplantation in children from living related donors. Surgical techniques and results. Ann Surg 214(4): 428-439.

4. Fan ST (2006) Live donor liver transplantation in adults. Transplantation 82(6): 723-732.

5. Song GW,LeeSG (2014) Living donorliver transplantation. Curr Opin Organ Transplant 19(3): 217-222.

6. Trotter JF, Adam R, Lo C, Kenison J (2006) Documented Deaths of Hepatic Lobe Donors for Living Donor Liver Transplantation 12: 1485-1488.

7. Sun Z, Yu Z, Yu S, Chen J, Wang J (2015) Post-Operative Complications in Living Liver Donors: A Single-Center Experience in China. Plos ONE 10(8): e0135557.

8. Morioka D, Egawa $\mathrm{H}$, Kasahara $\mathrm{M}$, Ito $\mathrm{T}$, Haga $\mathrm{H}$, et al. (2007) Outcomes of adult-to-adult living donor liver transplantation: A single institution's experience with 335 consecutive cases. Ann. Surg 245: 315-325.

9. Marsh JW, Gray E, Ness R, Starzl TE (2010) Complications of right lobe living donor liver transplantation. J Hepatol 51(4): 715-724.

10. Suh KS, Hong SK, Lee KW, Yi NJ, Kim HS, et al. (2018) Pure laparoscopic living donor hepatectomy: Focus on 55 donors undergoing right hepatectomy. Am J Transplant 18: $434-443$.

11. Kwon CHD, Choi GS, Kim JM, Cho CW, Rhu J, et al. (2018) Laparoscopic Donor Hepatectomy for Adult Living Donor Liver Transplantation Recipients. Liver Transplant 24: 1545-1553.

12. Agarwal S, Selvakumar N, Rajasekhar K, Dey R, Verma S, et al. (2019) Minimum absolute graft weight of $650 \mathrm{~g}$ predicts a good outcome in living donor liver transplant despite a graft recipient body weight ratio of less than 


\section{International Journal of Transplantation \& Plastic Surgery}

0.8. Clin. Transplant 33(10): e13705.

13. Albers MJ, de Gast-Bakker DA, van Dam NA, Madern GC, Tibboel D (2002) Male Sex Predisposes the Newborn Surgical Patient to Parenteral Nutrition-Associated Cholestasis and to Sepsis. Arch Surg 137(7): 789-793.
14. Kaplan M, Hammerman C, Vreman HJ, Wong RJ, Stevenson DK (2010) Hemolysis and hyperbilirubinemia in antiglobulin positive, direct $\mathrm{ABO}$ blood group heterospecific neonates. J Pediatr 157(5): 772-777.

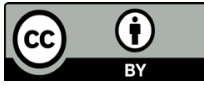

